\title{
EL APRENDIZAJE DE LA ESTADÍSTICA A TRAVÉS DE PBL CON FUTUROS PROFESORES DE PRIMARIA
}

\author{
Jon Anasagasti Aguirre \\ Ainhoa Berciano Alcaraz \\ Euskal Herriko Unibertsitatea/Universidad del País Vasco
}

\begin{abstract}
RESUMEN: Los contenidos estadísticos son un bloque fundamental de los currículos educativos que resultan indispensables en la toma de decisiones y, por tanto, ayudan en gran medida a desarrollar las competencias básicas y generales. A este respecto, investigaciones previas muestran que trabajar con proyectos o aprendizaje basado en problemas ( $\mathrm{PBL}$ ) usando métodos estadísticos y de probabilidad es un planteamiento didáctico satisfactorio, desarrollando tanto los conocimientos técnicos como los estratégicos. En este artículo se presenta el diseño de un módulo basado en PBL para trabajar esta disciplina con el alumnado del Grado de Educación Primaria justificando los instrumentos utilizados y valorando las aportaciones de cada uno.
\end{abstract}

PALABRAS CLAVE: Estadística, profesorado, educación primaria, aprendizaje basado en problemas.

\section{LEARNING STATISTICS THROUGH PBL WITH PROSPECTIVE PRIMARY TEACHERS}

\begin{abstract}
Statistical contents are a fundamental block in the curriculum that is essential to make decisions and, therefore, they help greatly to develop basic and general competences. On this point, previous researches show that working with projects or problem-based learning (PBL) using statistical and probability methods is a satisfactory teaching proposal, that develops both technical and strategic knowledge. In this article, the design of a PBL based module to work on this discipline with Teaching Degree (Primary Education) students is presented, justifying every tool used and evaluating the contribution of each one.

KEYWORDS: Statistics, teachers, elementary school, problem-based learning.
\end{abstract}

Recibido: 30/12/2014

Aceptado: 6/5/2015 
Correspondencia: Jon Anasagasti Aguirre, Departamento de Matemáticas y de las Ciencias Experimentales, Facultad de Ciencias y Tecnología de la Universidad del País Vasco, Apartado 664, 48080 Campus de Leioa, Bilbao. Email: jon.anasagasti@ehu.es.

\section{INTRODUCCIÓN}

El actual reto al que se enfrenta la educación estadística es ayudar a crear una sociedad culta en cuanto a la estadística ya que su uso se extiende a multitud de especialidades. Las disciplinas científicas tienden a acumular gran cantidad de información, pero sin los debidos procesos de recopilación, tratamiento y análisis se convierten inevitablemente en un inservible cúmulo de datos (Kettenrung, Lindsay y Siegmund, 2004).

Ante esta situación los distintos programas educativos incluyen la Estadística en sus currículos desde los estudios de Primaria hasta el Bachiller. Los alumnos de hoy día deben saber razonar de forma cuantitativa (Mertz, 2010) y para que esto sea posible sus maestros deben estar debidamente preparados.

Examinando estudios recientes sobre la evaluación del conocimiento estadístico de futuros profesores de Primaria, se recomienda mejorar la preparación estadística en cuanto al conocimiento especializado de la materia (Arteaga, Batanero y Cañadas, 2011). A pesar de que hoy día el alumnado esté familiarizado con gráficos estadísticos, tablas de doble entrada y conceptos como la media y la moda, siguen cometiendo muchos errores cuando se trata de entender el significado de nociones como la dispersión, la variabilidad o las posiciones relativas de los valores de centralización en distribuciones asimétricas (Anasagasti y Berciano, 2012). Pero no solamente ocurre esto con el conocimiento especializado; entre los futuros maestros de Primaria, gran parte del alumnado no relaciona los resultados del trabajo matemático con la situación problemática (Arteaga, Batanero y Ruiz, 2009).

La habilidad de aplicar los conocimientos matemáticos requiere del conocimiento técnico y del conocimiento estratégico. Dominar este último, saber cuándo hay que usar un concepto o un gráfico dado, es más difícil de lo que parece y en multitud de ocasiones suele ser obviado. Generalmente los problemas y ejercicios de los libros de texto escolares sólo suelen concentrarse en los conocimientos técnicos (Batanero y Díaz, 2011). Cuando se realiza un trabajo por proyectos, el alumnado se enfrenta a preguntas como ¿Cuál es el problema?, ¿Qué datos necesito?, ¿Cómo puedo obtenerlos?, ¿Qué entiendo de todo ello?

Cada vez son más las propuestas didácticas que tratan de trabajar el conocimiento, razonamiento o sentido estadístico a través de proyectos o por medio de resolución de problemas. Incluso nuevos materiales para Primaria fomentan este tipo de actividades que a través de la investigación y la realización de tareas, se acercan al conocimiento estadístico (Gil, 2010). Mediante este tipo de proyectos no sólo se consigue una mayor comprensión significativa, sino que además aparecen en el aula muchos conceptos teóricos que con ejercicios descontextualizados serían imposibles de apreciar como la variabilidad, la fiabilidad, la precisión o el sesgo.

Para alcanzar una comprensión matemática de calidad es necesario que las tareas propuestas estimulen el proceso cognitivo del estudiante. Así, países como Corea y 
Singapur, que mantienen un alto nivel competencial de matemáticas tal y como lo documentan los resultados PISA, mejoran también las habilidades de pensamiento crítico y resolución de problemas (Aizikovitsh-Udi, Clarke y Kuntze, 2014). Los nexos entre pensamiento estadístico y pensamiento crítico son evidentes.

\section{Problemática}

Existen cada vez más estudios acerca de la planificación e implementación de cursos específicos para la Didáctica de la Estadística entre futuros profesores y entre todos ellos abundan aquellos que promueven una enseñanza a través de escenarios, proyectos o el aprendizaje basado en problemas (PBL).

Se encuentran estudios en los que se fomenta la enseñanza de la Estadística a través de escenarios, promoviendo estrategias activas de aprendizaje, proyectos de colaboración, el uso de datos reales, simulaciones por ordenador y visualizaciones, con el fin de que los estudiantes adquieran una verdadera comprensión conceptual de los conceptos estadísticos (Azcárate y Cardeñoso, 2011). Este tipo de trabajos favorecen el aprendizaje significativo, promueven el trabajo en grupo y desarrollan capacidades como la reflexión y la autonomía del alumno, si bien la gestión del tiempo y del trabajo cooperativo, así como la organización novedosa de los contenidos supone una amenaza para el docente a la hora de preparar las sesiones.

En otros módulos se trabaja el contenido de la Estadística destacando el valor de esta para la resolución de problemas. En este caso los futuros profesores siguiendo las recomendaciones de Guidelines for Assessment and Instruction in Statistics Education (GAISE) se enfrentan a la resolución de problemas ateniéndose a cuatro componentes fijados en los Estándares para el Análisis de Datos y Probabilidad de la NCTM (Metz, 2010): Formulación de la pregunta; Recogida de datos; Análisis de datos; Interpretación de resultados.

Este tipo experiencias durante la Educación Primaria dan oportunidad al alumnado para aprender conceptos estadísticos más complejos cuando Ileguen a secundaria o a estudios superiores. En el caso de los futuros profesores de primaria además del conocimiento específico que han de poseer deben adquirir la competencia pedagógica necesaria para la enseñanza en el aula. Con la intención de que puedan adquirir conjuntamente el conocimiento técnico, el estratégico y el pedagógico se está intentando diseñar y poner en marcha un módulo de aprendizaje a través de PBL.

El propósito del estudio es valorar la mejora en las competencias estadísticas del alumnado propio de Magisterio mediante el aprendizaje basado en proyectos, trabajos grupales y resolución de problemas, y compararla con otro grupo que trabaje la estadística con metodología tradicional. Trabajar mediante proyectos con los futuros profesores puede ser muy valioso para que ellos mismos se sientan motivados a utilizar esta misma metodología con sus futuros alumnos de primaria.

En este caso concreto se presenta el módulo o método de trabajo seguido con un grupo de alumnos de la UPV/EHU, concretando los distintos instrumentos utilizados y viendo cuál es la utilidad de cada uno de ellos. Así mismo se pretende poner a juicio este método y sugerir los cambios que hagan que el módulo sea más efectivo. 


\section{Metodología}

Esta propuesta se encuentra enmarcada dentro de una investigación que pretende aportar los instrumentos útiles para una correcta adquisición de competencias para la docencia de la materia de Estadística y Probabilidad, y estudiar las ventajas obtenidas aplicando el módulo PBL en comparación con formas más tradicionales de aprendizaje. En este artículo se trata de mostrar la experiencia vivida por el alumnado durante el módulo Ilevado a cabo entre los meses de Enero y Febrero de 2014. Para ello se describen las distintas tareas realizadas durante el curso, se justifica el uso de cada una, se toman en cuenta las apreciaciones del profesor que lo ha impartido, y finalmente se recogen las opiniones dadas por el grupo de alumnos. Para recoger la opinión del alumnado se detallan las respuestas obtenidas en los distintos ítems del cuestionario de satisfacción (análisis cuantitativo) y se destacan los pareceres y sugerencias (análisis cualitativo) dadas por el alumnado.

Con todo ello se pretende evaluar críticamente el planteamiento y puesta en marcha del módulo, dejando para futuros trabajos la evaluación cuantitativa de los resultados que haya tenido dicho módulo sobre las competencias de futuros profesores.

\section{Participantes}

Participan en este módulo 69 de los 80 alumnos del grupo 32 de tercer curso del Grado en Magisterio de la Escuela de San Sebastián. Puesto que la participación era evaluativa y se requería la presencia obligatoria en el aula unos pocos alumnos optan por no participar si bien la mayor parte del grupo (86.25\%) accede a trabajar la materia mediante este módulo. Respecto al sexo de los participantes se encuentra que $47(68.1 \%)$ son mujeres y $22(31.8 \%)$ hombres. Ha de destacarse que se trata de un grupo que cursa la carrera en euskera y que todo el trabajo realizado se ha llevado a cabo en esta lengua.

\section{Método de Trabajo}

El bloque de "tratamiento de Datos Azar y Probabilidad" toma 1.5 créditos de los 9 que tiene la asignatura de Matemáticas y su Didáctica II y que se imparte dentro del Grado de Magisterio de Primaria. En cuanto al tiempo dedicado se limita a cinco semanas en las cuales se dispondrá en total para cada alumno de 6 horas de clases teóricas y 7 horas y media de clases prácticas.

Instrumentos de trabajo en el aula

A la hora de plantear un proyecto o módulo de trabajo es importante definir las actividades y materiales que se van a utilizar durante su transcurso ya que la debida preparación resulta imprescindible. En cursos como el descrito por Green y Blankenship (2013) utilizan principalmente actividades propuestas por CATALST, pero alrededor de esas actividades otro tipo de recursos como discusiones en aula, uso de software interactivo Tinkerplots ${ }^{\mathrm{TM}}$, conformar un diario de actividades o construir unidades didácticas, completan y enriquecen dicho curso. 
Distintos instrumentos de trabajo han sido utilizados durante este curso. Entre todos ellos se pretende que el alumnado pase por los tres niveles jerárquicos útiles para evaluar su conocimiento (Watson, 1997): que conozca los contenidos básicos de estadística y probabilidad, que comprenda los razonamientos y argumentos dentro de contextos más amplios y que desarrolle una actitud crítica para cuestionar argumentos basados en evidencias estadísticas.

En la Figura 1 se muestra la estructura del curso en el que se diferencian por un lado los dos instrumentos de presentación, los instrumentos tradicionales que se desarrollan principalmente durante las clases teóricas, y los distintos instrumentos que componen la puesta en marcha de un proyecto en el que el alumnado debe resolver un problema según las recomendaciones de la NCTM: Formulación de la pregunta, recogida de datos, análisis de datos e interpretación de resultados.

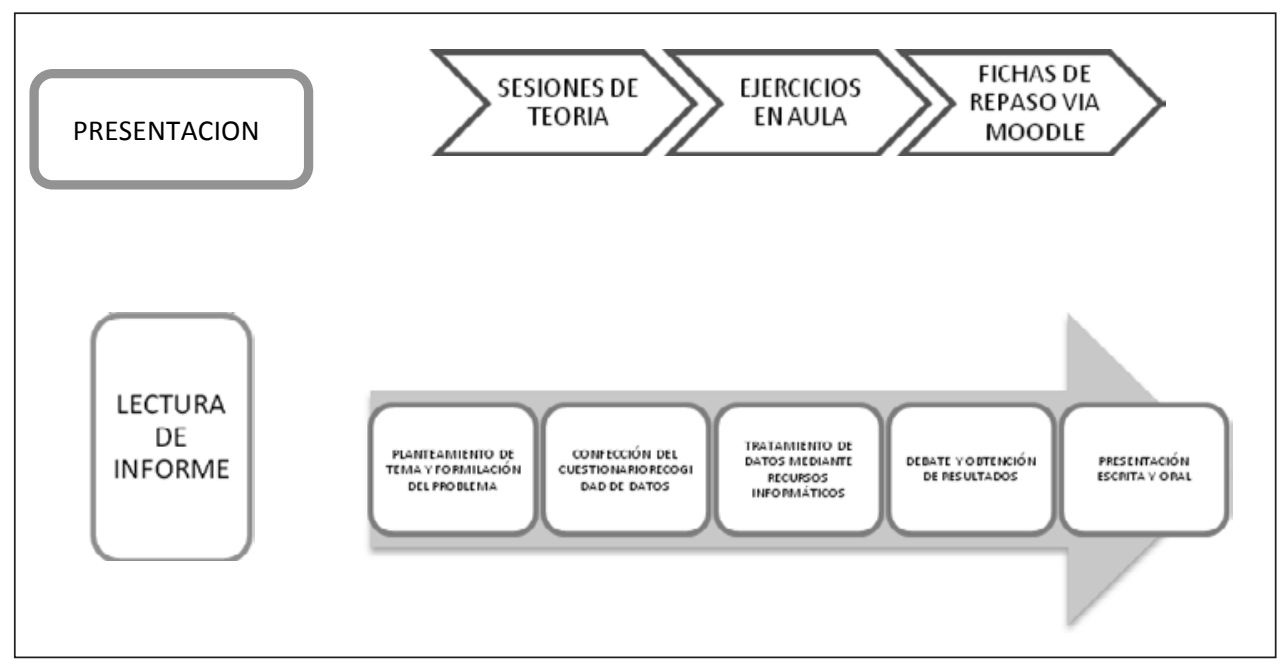

Figura 1. Estructura del curso

A continuación se detalla en qué consiste cada instrumento utilizado durante el curso, tanto en las sesiones teóricas como en las prácticas de aula.

\section{Presentación}

Esta primera parte se dedica a presentar la materia como ciencia. Se hace verbalmente por parte del profesor una pequeña introducción acerca del uso de las técnicas estadísticas a través de la historia y sus diversas aplicaciones hoy día. Además, se les pide que participen aportando campos en los que el uso de la estadística y la probabilidad sea habitual, asegurando que se mencionen la mayoría de las aplicaciones. El propósito de esta parte del módulo es que los alumnos tomen conciencia de la importancia que ha ido ganando esta disciplina en las últimas décadas, con el objetivo de que en sus futuras clases se trabaje la materia debidamente. 


\section{Sesiones de teoría}

Se trata de presentar al alumnado todos los conceptos necesarios para un corecto tratamiento de datos, el análisis de los experimentos aleatorios y el cálculo de probabilidades. Debido al reducido tiempo de que se dispone para la puesta en marcha del módulo se seleccionan los principales conceptos y se presentan en diversos documentos que están a su disposición a través de Moodle, y que están extraídos principalmente del Manual para el Estudiante del Proyecto Edumat-Maestros de "Estocástica y su Didáctica para Maestros" (Batanero, 2002).

\section{Ejercicios en aula}

Se presentan diversos ejercicios en los que deben tratar los datos presentados en tablas, para presentarlos en gráficas y viceversa. También deben calcular distintas medidas de centralización y de dispersión así como calcular probabilidades teóricas de distintos experimentos. Todos estos ejercicios tienen esencialmente la finalidad de trabajar los distintos conceptos desde el punto de vista numérico y de cálculo, así como de ser capaces de presentar los datos mediante diferentes recursos.

\section{Fichas de repaso vía Moodle}

Estas fichas tienen como objetivo el repaso de los conceptos trabajados en cada sesión teórica. Constan de cinco enunciados en los que deben señalar si son verdaderos o falsos; otros cinco enunciados en los que deben rellenar la parte vacía de la frase; y para finalizar uno o dos ejercicios acerca del tema tratado en la sesión. Cada alumno debe entregar a través de Moodle estas tres fichas antes de la siguiente sesión.

\section{Lectura de informe}

Se les ofrece la ocasión de reconocer los conceptos trabajados en el aula en un informe real proveyendo al contenido teórico un contexto cercano y familiar. Se trata de un informe publicado por la Dirección de Juventud del Departamento de Educación, Política Lingüística y Cultura del Gobierno Vasco. Es una investigación del Observatorio Vasco de la Juventud titulado "Lonjas y locales juveniles en la CAPV" que consta de una nota de prensa y una presentación de los datos cuantitativos. El objetivo es, que mediante trabajo grupal, reconozcan los distintos conceptos identificándolos en informes como los que muy probablemente tendrán que tratar en su futuro profesional. Para completar esta actividad los alumnos deben rellenar una ficha en la cual se les plantean preguntas acerca de la muestra del estudio, el tipo de datos ofrecidos, las gráficas utilizadas, las variables seleccionadas o las medidas de centralización utilizadas.

\section{Planteamiento de tema y problema}

Puesto que lo que se quiere conseguir es que el alumnado se implique en el trabajo, se les da la oportunidad de que ellos mismos elijan un tema para el que puedan realizar un estudio estadístico. Así mismo se les pide que una vez elegido el tema se 
planteen una pregunta concreta para la cual deberán dar respuesta, basándose en la información recogida mediante los cuestionarios. Se trata de que apliquen técnicas estadísticas a temas con los que se sientan identificados y que lo vean como un instrumento útil para respaldar sus opiniones.

\section{Elaboración de cuestionario}

Una vez definido el tema deben ser capaces de elaborar un cuestionario que se pasará al resto de compañeros. Con las respuestas recogidas deben conformar la información precisa y necesaria (limitándose a la muestra por conveniencia) para dar respuesta a sus planteamientos. Se les exige que por lo menos recojan dos variables cuantitativas con las que poder trabajar medidas de centralización como la media y la mediana, pero por lo demás se les da total libertad. Estos cuestionarios son enviados al profesor y tras las correcciones realizadas a sugerencia de este, se realizan copias para poder pasarlas en la siguiente sesión.

\section{Recogida de datos}

Durante este proceso cada grupo tiene la oportunidad explicar al resto de compañeros cómo han confeccionado el cuestionario: tipo de preguntas respuestas, variables, etcétera. Seguidamente se reparten las copias de los cuestionarios y cada alumno rellena el suyo. Mediante este proceso los distintos grupos comienzan a verbalizar los conceptos aprendidos y además reciben las opiniones y críticas constructivas al trabajo realizado cuando los demás compañeros expresan sus dudas acerca de las preguntadas planteadas por ellos.

\section{Tratamiento de datos}

Para el tratamiento de los datos el alumnado utiliza una hoja de cálculo que les facilite construir tablas de frecuencias, gráficas o realizar cálculos estadísticos. A pesar de que la muestra no sea de gran tamaño y pudiesen realizarlo de manera manual el conocimiento, aplicación y uso de las TIC en la materia resulta siempre de gran interés. Alumnado que hasta el momento no conocía dichos instrumentos ve su aplicabilidad y su uso no hace más que ampliar las competencias del futuro profesorado.

\section{Debate y resultados}

Una vez ordenados los datos y obtenidas las medidas de centralización y de dispersión cada grupo debate acerca de la respuesta que deben dar a la pregunta formulada al comienzo del estudio. Para ello analizan las respuestas obtenidas para cada pregunta y una vez que eligen los datos más significativos deciden cuál es su conclusión.

\section{Presentación escrita y oral}

En este último paso los distintos grupos deben plasmar en un póster las conclusiones a las que han Ilegado dando respuesta a su pregunta inicial. Para ello toman 
gráficas, tablas de frecuencia y datos significativos que apoyen su postura. Para terminar cada grupo presenta oralmente al resto de la clase la información que se ha recogido y las conclusiones a las que han Ilegado. Al finalizar la presentación el profesor formula varias preguntas a cada grupo acerca de los conceptos estadísticos y probabilísticos trabajados hasta el momento a las cuales los grupos u otros alumnos dan respuesta. Se trata de que expresen verbalmente los conocimientos adquiridos utilizando el lenguaje apropiado

\section{Material utilizado durante el curso}

Entre el material utilizado durante el curso destaca el Manual para el Estudiante del Proyecto Edumat-Maestros de "Estocástica y su Didáctica para Maestros" (Batanero, 2002) utilizado durante las explicaciones teóricas. Además de esto también se trabaja con una colección de ejercicios destinados a asimilar los conocimientos técnicos necesarios y el cálculo estadístico. Así mismo se crean las tres fichas que el alumnado debe completar y entregar a través de la plataforma Moodle con el fin de afianzar los contenidos tras cada una de las sesiones teóricas. También se ha dispuesto de las copias necesarias para trabajar el texto de "Lonjas y locales juveniles en la CAPV". Para terminar ha sido necesario realizar las copias de los distintos cuestionarios y conseguir un ordenador con software de hoja de cálculo para cada grupo.

\section{Papel del profesor}

El papel que toma el profesor durante estas sesiones varía considerablemente según el carácter de la tarea realizada. En este caso se pone la atención sobre el papel que el profesor desempeña principalmente en las sesiones prácticas. Mientras que durante las sesiones teóricas se requiere más protagonismo por parte del profesor, durante las sesiones prácticas en las que el alumnado trabaja en el proyecto su participación es mucho más discreta.

En este tipo de clases sus intervenciones consisten en exponer al principio de la sesión cuál va a ser el siguiente cometido que van a tener que llevar a cabo y explicar cómo pueden realizarlo. Los grupos trabajan de manera autónoma y el profesor atiende a sus preguntas a medida que surgen dudas, recordando y reforzando de esta manera los conceptos teóricos estudiados y planteando nuevos conceptos estadísticos. En el camino de conseguir mayores niveles de pensamiento, el profesor facilita un entorno donde conviven las actitudes de emulación con la de búsqueda autónoma, frustración y gratificación, aplicación y construcción, así como memoria y reflexión (Contreras, 1998). Todo ello conlleva una gran carga de trabajo para la preparación y puesta en marcha de las clases.

Otra de las labores más importantes por parte del profesor es la de concienciar al futuro profesorado de cómo se desarrolla la comprensión conceptual del propio alumnado, y por tanto una de sus obligaciones es hacerle comprender al futuro profesorado que la forma en la que ellos piensan será la forma en la pensará su futuro alumnado (Pfannkuch, 2008). 


\section{Opinión recogida por parte del alumnado}

Para comprobar el grado de satisfacción que muestran los alumnos se les ha pasado un cuestionario con ítems que pretenden evaluar la organización del módulo, el papel del profesor, la relación del módulo con los conceptos teóricos, la utilidad para su formación profesional y su propia participación. Este cuestionario recoge la opinión de 65 de los 72 participantes en el módulo.

Entre los resultados obtenidos se puede apreciar que la valoración general es bastante positiva, una media de 4.19 en una escala del 1 al 5. Entre los resultados más bajos encontramos los ítems "El trabajo grupal me ofrece facilidades y oportunidades que el profesor no puede darme" (3.83) y "El módulo ha ayudado a mejorar mis habilidades profesionales" (3.83). En cuanto los ítems mejor valorados han resultado ser "Me he encontrado a gusto durante el trabajo grupal" (4.65), "En general la valoración de este módulo es positivo" (4.45) y "Al preguntarle al profesor, las respuestas han sido claras y concretas" (4.43).

Al comparar las puntuaciones individuales se observa que los alumnos que peor han valorado el módulo lo hacen con un 3.71 mientras que los que mejor lo han valorado lo puntúan con una media de 4.93 siendo la desviación típica un 0.31.

En cuanto al estudio cualitativo en el que se muestran las opiniones del alumnado encontramos entre los más destacados los siguientes puntos:

Al preguntarles por qué es lo que más les ha interesado y les puede aportar sus respuestas han destacado el aprendizaje del manejo de la hoja de cálculo, expresar los datos mediante gráficas, manejar e interpretar los datos, preparar los cuestionarios, diseñar el proceso y poner en práctica una investigación:

"He aprendido a preparar el cuestionario, analizar los datos, y a representarlos mediante gráficas hechas con Excel" (Al.040). "Más que lo que hemos trabajado, me ha parecido interesante cómo lo hemos hecho; al fin y al cabo, han sido contenidos que hemos tenido que trabajar nosotros mismos" (Al.043). "La investigación realizada, ya que he aprendido de manera práctica" (AI.053). "Para trabajar las gráficas con los niños, interpretando los datos" (Al.017). "Que sea un trabajo basado en la vida real" (Al.027).

Al preguntarles sobre en qué les ha ayudado los debates en grupo responden mayoritariamente que les ha ayudado a comprender mejor los conceptos y a aclarar dudas, y a conocer la opinión e interpretación de los demás, y a colaborar para realizar el proyecto:

"A ver otros puntos de vista y completar el mío" (Al.022) "Me ha ayudado a apreciar conclusiones de los que yo no me había dado cuenta" (Al.025), "He aprendido a utilizar diferentes métodos" (Al.032). "A comprender mejor los conceptos teóricos" (Al.057). "Me ha ayudado a resolver las dudas" (Al.064).

Al preguntarles acerca de qué han aprendido al realizar la presentación oral se puede observar que ello refuerza el tener que interpretar los datos sacando conclusiones, aprender a exponerlos adecuadamente apreciando la riqueza que esconden y la dificultad que ello entraña, mejorar sus capacidades comunicativas. También les parecen muy interesantes los estudios, resultados y conclusiones que los demás grupos exponen ante la clase: 
Obtener conclusiones y contradicciones" (AI.015). "A improvisar y practicar las presentaciones orales" (Al.018). "Que no es fácil explicar al resto de una manera clara la información recogida y que lo mejor es ofrecerla de un modo general" (Al.027). "A examinar los datos profundamente y a sacar conclusiones" (AI.034). "Las distintas posturas de la sociedad acerca de diferentes temas" (Al.053).

Al preguntarles sobre qué es lo que mejorarían para el próximo curso gran parte de las respuestas coinciden en que debieran disponer de más tiempo, sobre todo para profundizar más en el proceso de investigación. También solicitan mayores explicaciones sobre cómo usar los programas de hoja de cálculo y en algún caso indican que durante el trabajo en grupo en algunas tareas solamente uno o dos compañeros del grupo estaban ocupados.

"Disponer de más tiempo y poder pasar el cuestionario a más gente" (Al.019). "Trabajar más el uso del Excel" (Al.032). "Más tiempo para poder realizar una investigación más completa" (Al.034). "Trabajar de forma más activa; las sesiones teóricas de 2 horas dan mucha facilidad para desconectar" (Al.039). "Dejaría mayor libertad" (Al.048). "Acortar las clases teóricas" (Al.063).

De todas las respuestas dadas por el alumnado se puede concluir que el proceso de investigación les resulta atractivo y motivador, principalmente por enseñarles nuevos métodos de trabajo e iniciarles en el uso de herramientas informáticas que hasta ese momento raramente habían utilizado. El trabajo en grupos ha generado discusiones que han sido útiles para aclarar dudas y compartir conocimientos. Para terminar, tener que realizar las presentaciones les ha exigido mayor concentración y desarrollar su la capacidad para seleccionar información significativa, así como desarrollar sus competencias comunicativas.

\section{Evaluación de los conocimientos adquiridos}

Este apartado se presenta como uno de los mejores instrumentos de los que se dispone para comprobar la efectividad del módulo trabajado. Para poder evaluar la progresión y la asimilación de competencias los futuros profesores realizaron un pretest en el que se evaluaban las distintas competencias que recoge la guía de Grado de Educación primaria de la UPV/EHU.

Este mismo cuestionario ha sido utilizado en anteriores estudios como en las dos pruebas exploratorias sobre competencias de futuros Maestros de primaria: conocimiento de conceptos básicos de estadística (Anasagasti y Berciano, 2012) y conocimiento del bloque relativo al tratamiento de la información, azar y probabilidad, en el currículo escolar de matemáticas (Anasagasti y Berciano, 2013) donde se pretendían evaluar esas competencias. En esta ocasión, tras las necesarias correcciones realizadas en el texto del cuestionario se han vuelto a pasar en el pre-test y se volverán a pasar cuando los alumnos vuelvan de su periodo de Prácticas. De esta manera se ha querido dejar un espacio de tiempo suficiente para comprobar que las competencias alcanzadas son realmente interiorizadas, y no solamente las alcanzadas al término de las sesiones preparadas y recién trabajadas.

Es por esta razón por la que aún no disponemos de los resultados del post test por lo que esos resultados serán discutidos en futuros trabajos. 


\section{Discusión DE RESULTADOS}

Este trabajo se enmarca dentro de uno más amplio que analizará los datos sobre la progresión que el grupo de estudiantes ha experimentado sobre sus competencias acerca de la estadística. De todas maneras durante el desarrollo del módulo se ha podido apreciar cualitativamente que la motivación e implicación por parte del alumnado ha aumentado considerablemente.

Muchos de los estudios previos que fomentan el aprendizaje mediante ABP o realización de proyectos concluyen que trabajar con datos del contexto del alumnado no solo favorece su "motivación" sino que hace más comprensible las distintas explicaciones acerca de conceptos abstractos. Además también consigue que se muestren algunos conceptos que de forma teórica o por medio de ejercicios descontextualizados difícilmente podrían observarse.

Por medio de todos estos instrumentos se ha tratado de que todos los alumnos pasen por los tres niveles jerárquicos definidos por Watson (1997), aprendiendo los contenidos básicos de estadística, siendo capaces de razonar y argumentar dentro de contextos más amplios y desarrollando una actitud crítica basada en evidencias estadísticas.

Todo el proceso debe estar debidamente planificado ya que el aprendizaje no se logra únicamente en las sesiones en las que ofrecen conclusiones o definiciones. Es importante mencionar que aunque la tendencia sea poner toda la atención en los datos, lo verdaderamente importante es concretar la cuestión inicial para la cual todos estos datos serán utilizados con el fin de darle una respuesta (Friel, O'Connor y Mamer, 2006). Desde el principio del proceso el alumnado ha de ser capaz de proponer su propio problema, siendo ellos mismos los que inician el PBL. Posteriormente mediante la recogida de datos y utilizando los métodos estadísticos deben ser capaces de dar la respuesta.

Profundizar en los distintos estudios propuestos por los alumnos resulta en ocasiones complicado debido al reducido tiempo del que se dispone para trabajar la asignatura y más en concreto esta parte. No obstante el objetivo del módulo PBL al margen de transmitir los conceptos estadísticos necesarios es motivar al estudiante a utilizar métodos de investigación, tratar los datos con instrumentos informáticos, analizar la información y mostrar sus conclusiones a partir de estos. Al margen de las mejoras que se puedan realizar en todo el proceso, se concluye que todos estos objetivos se han logrado gracias al trabajo realizado en el módulo PBL.

\section{REFERENCIAS BIBLIOGRÁFICAS}

Aizikovitsh-Udi, E., Clarke, D., y Kuntze, S. (2014). Hybrid tasks: Promoting statistical thinking and critical thinking through the same mathematical activities. $P N A, 8(3)$, 115-126.

Anasagasti, J., y Berciano, A. (2012). Prueba exploratoria sobre competencias de futuros maestros de primaria: conocimiento de conceptos básicos de estadística. En Investigación en Educación Matemática XVI (SEIEM), pp. 113-121.

Anasagasti, J., y Berciano, A. (2013). Prueba exploratoria sobre competencias de futuros maestros de primaria: Conocimiento del bloque relativo al tratamiento de 
la información, azar y probabilidad, en el currículo escolar de Matemáticas. En J. M. Contreras, G. R. Cañadas, M. M. Gea y P. Arteaga (Eds.), Actas de las Jornadas Virtuales en Didáctica de la Estadística, Probabilidad y Combinatoria (pp. 531538). Granada: Departamento de Didáctica de la Matemática de la Universidad de Granada.

Arteaga, P., Batanero, C., y Cañadas, G. (2011). Evaluación del conocimiento especializado de la estadística en futuros profesores en una tarea abierta. En M. Marín Rodríguez, G. Fernández García, L. J. Blanco Nieto y M. Palarea Medina (Eds.), Investigación En Educación Matemática XV (pp. 267-276). Ciudad Real: Sociedad Española de Investigación en Educación Matemática, SEIEM.

Azcárate, P., y Cardeñoso, J.M. (2011). La Enseñanza de la Estadística a través de Escenarios: implicación en el desarrollo profesional. Bolema, 24(40), 789-810.

Batanero, C. (2013). Sentido estadístico: Componentes y desarrollo. Actas de las $1^{a}$ Jornadas Virtuales en Didáctica de la Estadística, Probabilidad y Combinatoria, SEIEM. (Junio 2013) Número I, Año I, vol. 2.

Batanero, C., Díaz, C., Contreras, J. M., y Arteaga P. (2011). "Enseñanza de la estadística a través de proyectos". En C. Batanero y C. Díaz (Eds.), Estadística con proyectos. Granada: Universidad de Granada.

Batanero, C., y Godino, J. D. (2002). Estocástica y su didáctica para maestros. Granada: Universidad de Granada, Departamento de Didáctica de la Matemática.

Contreras, L. C. (1998). Resolución de problemas: un análisis exploratorio de las concepciones de los profesores acerca de su papel en el aula (Tesis Doctoral, Universidad de Huelva).

DECRETO 97/2010, de 30 de marzo, por el que se modifica el Decreto que establece el currículo de la Educación Básica y se implanta en la Comunidad Autónoma del País Vasco (BOPV, 20 de Abril de 2010). Recuperado de http://www.euskadi.eus/ bopv2/datos/2010/04/1002109a.pdf.

Friel, S., O'Connor, W., y Mamer, J. (2006). More than "Meanmedianmode" and a bar graph: What's needed to have a statistical conversation? En G. Burrill y P. C. Elliott (Eds.), Thinking and reasoning with data and chance (pp. 117-137). Reston, Va: National Council of Teachers of Mathematics.

Gil, A. R. (2010). Proyectos de Estadística en Primaria. Material editado por el Instituto Canario de Estadística (ISTAC). Números. Revista de Didáctica de las Matemáticas, 75, 121-129.

Godino, J. D., Batanero, C., Rivas, H., y Arteaga, P. (2013). Componentes e indicadores de idoneidad de programas de formación de profesores en didáctica de las matemáticas. REVEMAT, 8(1), 46-74.

Green, J. L., y Blankenship, E. E. (2013). Primarily statistics: Developing an introductory statistics course for pre-service elementary teachers. Journal of Statistics Education, 21(3).

Lindsay, B. G., Kettenring, J., y Siegmund, D. O. (2004). A report on the future of Statistics. Statistical Science, 19(3), 387-413.

Metz, M. L. (2010). Using GAISE and NCTM standards as frameworks for teaching probability and statistics to pre-service elementary and middle school mathema- 
tics teachers. Journal of Statistics Education, 18(3). Recuperado de http://search. proquest.com/docview/851224164?accountid=17248.

Pfannkuch, M. (2008). Training teachers to develop statistical thinking. En C. Batanero, G. Burrill, C. Reading y A. Rossman (Eds.), Joint ICMI/IASE Study: Teaching Statistics in School Mathematics. Challenges for Teaching and Teacher Education. Proceedings of the ICMI Study 18 and 2008 IASE Round Table Conference. [Dordrecht, etc.]: Springer. doi: 10.1007/978-94-007-1131-0.

Strangfeld, J. A. (2013). Promoting active learning: Student-led data gathering in undergraduate statistics. Teaching Sociology, 41(2), 199-206.

Watson, J. (1998). Assessment of statistical understanding in a media context. ICOTS 5, Research in Teaching statistics, 793-799. 
